\title{
Alter
}

Revue de phénoménologie

$25 \mid 2017$

L'Histoire

\section{Une réponse husserlienne? Réfutation et absorption des objections heideggériennes dans les manuscrits de Husserl}

Julien Farges

\section{OpenEdition}

Journals

Édition électronique

URL : http://journals.openedition.org/alter/416

DOI : $10.4000 /$ alter.416

ISSN : 2558-7927

Éditeur :

Association ALTER, Archives Husserl (CNRS-UMR 8547)

\section{Édition imprimée}

Date de publication : 3 décembre 2017

Pagination : 215-233

ISBN : 978-2-9550449-3-3

ISSN : 1249-8947

Référence électronique

Julien Farges, « Une réponse husserlienne ? Réfutation et absorption des objections heideggériennes dans les manuscrits de Husserl », Alter [En ligne], 25 | 2017, mis en ligne le 01 décembre 2018,

consulté le 02 mai 2019. URL : http://journals.openedition.org/alter/416 ; DOI : 10.4000/alter.416 


\title{
UNE RÉPONSE HUSSERLIENNE ? RÉFUTATION ET ABSORPTION DES OBJECTIONS HEIDEGGERIENNES DANS LES MANUSCRITS DE HUSSERL
}

\begin{abstract}
Julien Farges
Il ne prouve que lui-même, son unique preuve est lui-même, tous les adversaires le vainquent aussitôt mais ce n'est pas qu'ils le réfutent (il est irréfutable), c'est qu'ils se prouvent eux.

Franz Kafka
\end{abstract}

Si une meilleure connaissance de la pensée du jeune Heidegger a permis de faire apparaître Sein und Zeit au moins autant comme un point d'aboutissement que comme un point de départ, et de battre en brèche l'idée selon laquelle l'ouvrage devrait l'essentiel de sa sub-

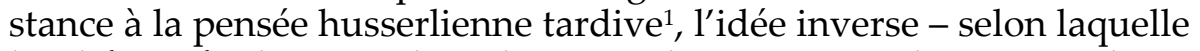
la phénoménologie tardive de Husserl et en particulier ses analyses centrées sur le monde de la vie seraient globalement reconductibles à une influence de Heidegger - semble encore avoir cours. Sans prétendre épuiser les enjeux de la controverse entre les deux philosophes, la présente étude souhaite contribuer à sa meilleure compréhension moyennant la critique de cette dernière conception, de sorte que soit écartée toute interprétation en termes d'influence de l'un des deux philosophes sur l'autre. En proposant une reconstruction de la « réponse transcendantale » que les manuscrits husserliens élaborent

\footnotetext{
${ }^{1}$ On reconnaît la lecture proposée par M. Merleau-Ponty dans l'Avant-propos de la Phénoménologie de la perception, Paris, Gallimard, 1945, p. I : «[...] tout Sein und Zeit est sorti d'une indication de Husserl et n'est en somme qu'une explication du "natürlichen Weltbegriff" ou du [sic] "Lebenswelt" que Husserl, à la fin de sa vie, donnait pour thème premier à la phénoménologie [...] ». Pour une mise en perspective de Sein und Zeit avec les axes principaux de la philosophie du jeune Heidegger, on consultera notamment Heidegger 1919-1929. De l'herméneutique de la facticité à la métaphysique du Dasein, J.-F. Courtine (éd.), Paris, Vrin, 1996.
} 
face à la conception de phénoménologie exposée dans Sein und Zeit, il $\mathrm{s}^{\prime}$ agit plutôt d'étayer la conception selon laquelle cette dernière, par les objections qu'elle adresse à la phénoménologie transcendantale, a sollicité et comme contraint Husserl à une clarification de certains de ses principes et concepts fondamentaux, clarification qui s'est traduite par un approfondissement de leur portée philosophique et de leurs ressources descriptives eu égard notamment à la sphère thématique de l'existence humaine pratique et quotidienne. Ainsi la réponse husserlienne à Heidegger coïnciderait-t-elle avec une réappropriation de la phénoménologie transcendantale par elle-même, avec le développement d'une " phénoménologie de la phénoménologie ».

\section{La triple critique heideggérienne}

Il est bien connu que les objections que Heidegger adresse à la phénoménologie husserlienne - notamment dans le contexte de la rédaction commune de l'article "Phénoménologie » destiné à l'Encyclopædia Britannica en $1927^{2}$ - gravitent toutes autour de l'unique problème du mode d'être de la subjectivité transcendantale. L'argument directeur est le suivant : si la phénoménologie constitutive élucide bien le sens d'être du monde et de tout étant mondain dans la mesure où elle le renvoie aux effectuations constituantes d'une subjectivité transcendantale, elle n'en laisse pas moins dans l'ombre le mode d'être de cette instance constituante elle-même ${ }^{3}$. Or les conséquences de cette lacune - qui relève moins d'un oubli contingent que $\mathrm{d}^{\prime}$ un manquement (Versäumnis) nécessaire ${ }^{4}$ - dépassent largement la sphère de la seule égologie, puisqu'elles conduisent à une mise en

\footnotetext{
${ }^{2}$ L'ensemble est publié in E. Husserl, Phänomenologische Psychologie. Vorlesungen Sommersemester 1925, La Haye, Martinus Nijhoff, 1962 (désormais : PP), Hua IX, resp. p. 239-253, p. 271-277 et p. 601-602 ; trad. J.-L. Fidel et J.-F. Courtine in E. Husserl, Notes sur Heidegger, Paris, Minuit, 1993, resp. p. 78-91, p. 108-114 et p. 117-118. Pour une présentation du dossier et de ses enjeux, cf. B. Hopkins, "The Husserl-Heidegger Confrontation and the Essential Possibility of Phenomenology », in Husserl Studies, n 17, 2001, p. 125-148 ; S. G. Crowell, Husserl, Heidegger and the Space of Meaning. Paths towards Transcendental Phenomenology, Evanston, Northwestern University Press, 2001, p. 174 sq. Pour une mise en perspective globale des deux projets phénoménologiques et une bibliographie détaillée sur la question, cf. S. Overgaard, Husserl and Heidegger on Being in the World, Dordrecht, Kluwer Academic Publishers, Phaenomenologica 173, 2004.

${ }^{3}$ Cf. M. Heidegger, in E. Husserl, PP, Hua IX, p. 601-602 ; trad. cit. (modifiée), p. 117-118 : «le problème qui se pose immédiatement est de savoir quel est le mode d'être de l'étant dans lequel le "monde" se constitue. [...] Quel est le mode d'être de cet ego absolu ? En quel sens est-il identique au Moi toujours facticiel, en quel sens est-il différent? ".

${ }^{4}$ Cf. M. Heidegger, Prolegomena zur Geschichte des Zeitbegriffs, von P. Jaeger (éd.), Francfort-surle-Main, Vittorio Klostermann, 1994, GA 20, p. 157 ; trad. A. Boutot, Prolégomènes à l'histoire du concept de temps, Paris, Gallimard, 2006, p. 170.
} 
question globale de la phénoménologie husserlienne se déployant sous la forme d'un double reproche d'abstraction et de théoricisme.

Pour comprendre la portée de ce double reproche, il convient tout d'abord de préciser que pour être phénoménologiquement insatisfaisant, le concept de subjectivité transcendantale n'est pas pour autant absurde ni vide aux yeux de Heidegger ; simplement ne tient-il pas sa compréhensibilité ni sa légitimité de lui-même. C'est ce que veut dire Heidegger lorsqu'il suggère à Husserl d'interpréter la réduction phénoménologique non plus comme déshumanisation de l'ego mais bien comme réalisation d'une essentielle possibilité de l'existence humaine ${ }^{5}$. Cela signifie en fin de compte que c'est du Dasein que l'ego transcendantal reçoit sa signification philosophique, ou encore que cet ego transcendantal doit être pensé comme l'une des possibilités existentiales du Dasein. Par conséquent, s'il est considéré séparément du tout existential concret au sein duquel il reçoit son sens, il se réduit à une vue abstraite prise sur l'homme, et la philosophie qui l'isole méthodiquement de ce tout se rend elle-même coupable d'abstraction. Si la phénoménologie de la constitution transcendantale (Konstitution) a un sens, ce n'est donc que dans la mesure où est reconnue sa dépendance relativement à une phénoménologie qui rendra compte de la « constitution (Verfassung) existentielle du Dasein $»^{6}$. Il ne s'agit donc pas de substituer la Verfassung existentiale à la Konstitution transcendantale, mais de faire de l'élucidation de celle-ci une tâche subordonnée dans le cadre de l'élucidation de celle-là. Or, ainsi éclairci, ce reproche d'abstraction mène directement à celui de théoricisme.

En effet, si l'on se demande quelle est la possibilité existentiale du Dasein concret qui correspond à la libération réductive de l'ego transcendantal, on est immédiatement renvoyé à la connaissance, comme mode sur lequel un «sujet » tente de conquérir théoriquement la transcendance d'un « objet » ou du monde lui-même comme totalité objective. Or du point de vue de l'analytique existentiale, la connaissance du monde, loin d'être le mode originaire de l'être-au-monde, n'est qu'un mode dérivé de l'être à... comme tel. Le rapport originaire de l'homme au monde consiste en un être-auprès-du monde relevant d'un engagement pratique dont le sens renvoie quant à lui au souci (Sorge) comme détermination ontologique fondamentale du Dasein.

\footnotetext{
${ }^{5}$ Cf. M. Heidegger, in E. Husserl, PP, Hua IX, p. 275, note 1 (trad. cit., p. 111, note 6 : « Ou peutêtre suis-je [sc. dans l'exercice de la réduction à mon propre égard] justement un Je dans sa possibilité d'existence la plus propre et la plus "merveilleuse" ») et p. 277, note 1 (trad. cit., p. 113, note 7 : « une possibilité humaine dans laquelle l'homme justement arrive à lui-même »).

${ }^{6}$ M. Heidegger, in E. Husserl, PP, Hua IX, p. 601-602 ; trad. cit., p. 117.
} 
Dans ces conditions, c'est le concept de Zuhandenheit qui s'impose pour rendre compte de la façon dont le monde s'annonce à la circonspection du Dasein. Or, comme le souligne Gadamer, «ce que Heidegger [nous] a appris avec le primat de la Zuhandenheit [...] contredit l'ensemble de l'ordre d'édification et de fondation des intentionnalités qui est établi dans la phénoménologie constitutive de Husserl $»^{7}$. De fait, au sein de cet ordre stratifié, c'est la simple nature perceptive qui fonctionne comme couche fondamentale de la réalité, c'est-à-dire comme infrastructure de toute Umwelt concrète et signifiante possible. Or c'est très précisément ce qui se trouve renversé par l'élucidation ontologico-existentiale du phénomène du monde, si bien que le sens originaire du phénomène du monde n'est autre, dans la perspective heideggérienne, que celui qui vient en dernier lieu dans l'édification stratifiée de la réalité chez Husserl - 1'Umwelt quotidienne, pratique et personnaliste, lestée de prédicats historico-culturels idéaux et ouverte à la compréhension intersubjective. Ce sens originaire du monde ambiant, la phénoménologie constitutive husserlienne semble donc condamner à le manquer.

Face à cette triple critique (manquement relatif au mode d'être de l'instance constituante, abstraction et théoricisme de la phénoménologie constitutive), les commentateurs ont souvent été tentés de montrer qu'elle fait bon marché de telle ou telle analyse ou mise au point locale de Husserl et qu'elle n'est donc que partiellement justifiée ${ }^{8}$. Mais on a rarement pris en compte la réaction proprement philosophique de Husserl telle qu'elle se déploie le plus souvent dans les manuscrits de recherche contemporains de sa confrontation littérale avec les travaux de Heidegger; or leur étude montre non seulement que Husserl a pris la mesure des objections que lui adresse son ancien étudiant, mais qu'il s'efforce, en particulier entre 1930 et 1931, d'orchestrer en retour quelque chose comme une "réponse transcendantale ». Nous allons montrer à présent que cette défense et illustration de la phénoménologie constitutive transcendantale, telle qu'elle s'élabore dans les manuscrits de recherche, se laisse reconstruire à partir de l'alternative entre la réfutation et l'absorption, chacune des deux pre-

\footnotetext{
${ }^{7}$ H.-G. Gadamer, «Die phänomenologische Bewegung », in Philosophische Rundschau, vol. 11, 1963, p. $1-45$; ici p. 37.

${ }^{8}$ Cf. par exemple R. Hickerson, "Neglecting the Question of Being : Heidegger's Argument against Husserl », in Inquiry, An International Journal of Philosophy, [en ligne], vol. 52, n 6, 2009, p. 574-595; de façon plus générale, cf. également T. J. Stapleton, Husserl and Heidegger: The Question of a Phenomenological Beginning, Albany, State University of New York Press, 1983 - qui interprète la phénoménologie husserlienne comme un projet ontologique; B. Hopkins, Intentionality in Husserl and Heidegger: The Problem of the Original Method and Phenomenon of Phenomenology, Dordrecht, Kluwer Academic Publishers, 1993.
} 
nant à son tour deux formes. La réfutation signifie de la part de Husserl soit la pure et simple dénégation, soit, plus subtilement, le retournement de l'objection contre son auteur. Quant à l'absorption, elle s'accomplit soit sous la forme de la neutralisation de l'objection par son intégration à l'analyse intentionnelle, soit sous la forme de la réélaboration par Husserl de certains de ses concepts fondamentaux à partir de ce que Heidegger a fait valoir contre sa phénoménologie. C'est dans ce dernier cas seulement qu'il serait alors légitime de parler $d^{\prime}$ « influence » du disciple sur le maître - étant bien entendu qu'elle s'effectue toutefois, du point de vue de Husserl, au bénéfice exclusif et intégral du transcendantalisme.

\section{La dénégation du théoricisme et la question de la primitivité de l'analyse phénoménologique}

Commençons par l'objection de théoricisme, dont on peut dire qu'elle fait apparemment l'objet d'une simple dénégation de la part de Husserl, en particulier lorsqu'il revient, dans un manuscrit du mois de mai 1931, sur la mise en évidence de la structure esthétique du monde comme première strate constitutive de ce dernier :

Lorsqu'on commence, comme je le fais, par dégager à la façon d'une esthétique transcendantale un concept naturel de monde, cela désigne, comme je le pense encore en dépit de Heidegger, un système de tâches nécessaires et premier en soi, et cela signifie que je n'ai fait que choisir autrement le cours de la considération théorique abstractive, mais d'une certaine façon plus primitivement que ne l'a fait Heidegger'.

Que l'élaboration d'un concept naturel de monde soit une tâche nécessaire et première en soi, Heidegger ne le contesterait pas puisqu'il affirme au contraire, dans le $\S 11$ de Sein und Zeit, qu'il s'agit $d^{\prime}$ une exigence incluse dans le point de départ de l'analytique existentiale ${ }^{10}$. Le différend porte plutôt ici sur la question de savoir si une esthétique transcendantale phénoménologique est à même de satisfaire à une telle exigence. Du point de vue de Heidegger, une telle identification du monde primordial à un monde esthétique relève du

\footnotetext{
${ }^{9}$ E. Husserl, Zur phänomenologischen Reduktion. Texte aus dem Nachlass (1926-1935), Dordrecht, Kluwer, 2002 (désormais : ZPR), Hua XXXIV, p. 260 ; trad. J.-F. Pestureau, De la réduction phénoménologique. Textes posthumes (1926-1935), Grenoble, Jérôme Millon, 2007, p. 238 (intégralement retraduit).

${ }^{10}$ Cf. M. Heidegger, Sein und Zeit, Tübingen, Niemeyer, 1976, p. 50 sq. ; trad. E. Martineau, Être et temps, Paris, Authentica, 1985, p. 59 sq.
} 
plus pur théoricisme dans la mesure où c'est seulement au regard théorique que le monde se manifeste comme nature perceptive, c'està-dire comme totalité spatio-temporelle et in-signifiante des corps physiques. Cela dit, Husserl semble soutenir dans ces lignes que la perspective heideggérienne se distingue de la sienne seulement par le degré : l'une et l'autre seraient commandées par l'attitude théorique, mais celle de Husserl le serait plus primitivement. Comment le comprendre?

En commençant par insister sur le fait que Husserl se montre parfaitement conscient non seulement du caractère théorique de son analyse structurale du phénomène du monde, mais aussi de ce que l'attitude théorique n'est pas l'attitude dominante de la vie humaine concrète. Ainsi écrit-il dans un autre manuscrit, contemporain du précédent :

Il peut bien se faire, et il en va bien sûr de la sorte, qu'un intérêt théorétique et une effectuation théorétique ne soient qu'une ligne de conduite dans la vie humaine et pas du tout dans toute vie humaine. Mais devons-nous être dans une autre attitude, nous qui méditons ici, puisque nous posons bien des questions concernant la vérité11 ?

Toute la question est donc de savoir si le phénoménologue peut, en tant que philosophe interrogeant le phénomène du monde et l'expérience du monde en leur vérité, adopter une autre attitude que l'attitude non pratique de la théorisation. Qu'il y ait des vérités pratiques est hors de doute ; mais leur reconnaissance comme vérités peut-elle ne pas s'effectuer théoriquement? Pour Husserl, le phénoménologue est condamné, s'il s'agit bien pour lui de philosopher et non de distraire, d'édifier ou de se livrer à de la poésie conceptuelle ${ }^{12}$, à adopter la posture théorique qui convient à toute recherche de quelque chose comme une vérité, à se soumettre par conséquent à un ordre des généralités qui n'est pas celui de la vie pratique concrète. Dans ces conditions, il suffit de reconnaître que «pour l'homme théorique, la structure pratique universelle du monde de la vie en sa généralité $\mathrm{n}^{\prime}$ est pas ce qui est premier $»^{13}$. Autrement dit, $c^{\prime}$ est la distinction entre ce qui est premier en soi et ce qui est premier pour nous qui permet d'échap-

\footnotetext{
${ }^{11}$ E. Husserl, ZPR, Hua XXXIV, p. 257-258 ; trad. cit. (modifiée), p. 236.

${ }^{12}$ Cf. la pointe sur les «poètes du concept » (in E. Husserl, Die Krisis der europäischen Wissenschaften und die transzendentale Phänomenologie. Eine Einleitung in die phänomenologische Philosophie, La Haye, Martinus Nijhoff, 1954 (désormais : Krisis), § 57, Hua VI, p. 204 ; trad. G. Granel, La crise des sciences européennes et la phénoménologie transcendantale, Paris, Gallimard, 1976, p. 227), qui vient relayer l'allusion aux "gens de lettres de la philosophie » (ibid., §7, Hua VI, p. 15; trad. cit., p. 22).

${ }^{13}$ E. Husserl, ZPR, Hua XXXIV, p. 261 ; trad. cit. (modifiée), p. 239.
} 
per au reproche de théoricisme en rendant compatibles une fondation esthétique du monde réal et la reconnaissance de la primauté concrète du monde pratique :

Qu'est-ce qui, pour l'intérêt théorique éveillé, est premier, ou peut l'être ? La structure du monde pratique environnant et du moi, ou du nous comme pratiques, n'est-elle pas pour lui quelque chose de très tardif, quoique indubitablement le monde pratique soit le plus concret en corrélation avec la subjectivité pratiquement intéressée, vivant conscientiellement de façon pratique ${ }^{14}$ ?

Reprocher à la phénoménologie transcendantale son théoricisme revient donc à lui reprocher de se vouloir une philosophie plutôt qu'une vision du monde. Le caractère pleinement constituant de la subjectivité pratique $\mathrm{s}^{\prime} \mathrm{y}$ trouve parfaitement reconnu et établi, mais seulement à la place qui lui revient dans l'ordre imposé par l'exigence philosophique d'un discours visant la validité universelle ${ }^{15}$.

Si l'objection heideggérienne semble ainsi seulement écartée, il ne faut pas méconnaître que les propos de Husserl construisent simultanément, en sous-main, une objection à l'encontre de l'analytique existentiale. L'argument est simple: si elle prétend établir descriptivement la vérité du sens d'être de l'homme et de son rapport avec la question de l'être et celle de la vérité elle-même, s'il y va d'autre chose en elle que d'une simple vision du monde, alors la phénoménologie heideggérienne doit nécessairement relever elle aussi, quoique Heidegger en dise, d'une attitude théorique, si bien que ce qu'il reproche à la phénoménologie transcendantale doit nécessairement se retrouver à l'œuvre dans sa propre démarche. En d'autres termes, celle-ci a beau procéder de façon directe et non explicitement réductive, il n'en reste pas moins

qu'une telle démarche, comme toute démarche scientifique au sens le plus large, est une démarche [accomplie dans un] intérêt théorique conséquent, qui livre donc ses résultats en ce qu'à la naturalité de la vie pratique de niveau pré-théorétique se superpose une attitude théorétique, celle d'un spectateur "non engagé », dirigé purement sur ce qui est là, lequel, sans être lui-même impliqué en cette praxis, la regarde, médite sur elle, suit ses genres et [ses] formes, sa motivation, ses niveaux de formation et de signification ${ }^{16}$.

\footnotetext{
14 Ibid., Hua XXXIV, p. 260 ; trad. cit. (modifiée), p. 238 (nous soulignons).

${ }^{15} \mathrm{Cf}$. idem : «La subjectivité pratique n'est-elle pas aussi constituante, la démarche de ma phénoménologie s'est-elle jamais contentée de dégager constitutivement la nature propre au chercheur en sciences de la nature? ».

16 Ibid., Hua XXXIV, p. 259 ; trad. cit. (modifiée), p. 237.
} 
Il pourrait être tentant de voir en cette déclaration le comble de la mécompréhension husserlienne de la démarche de Sein und Zeit, puisque le rejet de l'idée même d'un spectateur désengagé est, chez Heidegger, une composante essentielle de cette critique de la subjectivité auto-réflexive dont procède la mise en avant du concept de Dasein, et puisque Husserl semble ne pas tenir compte de la redéfinition du concept de vérité proposée par Heidegger dans le cadre d'une phénoménologie renouvelée par l'herméneutique. C'est indéniable, mais il n'est pas sûr que cela change grand chose si ce que Husserl vise en s'attaquant au discours tenu par Heidegger dans l'ouvrage de 1927, c'est l'absence en lui d'une justification existentiale de sa propre possibilité. Dans ces conditions, la contre-objection husserlienne consiste en fin de compte à diagnostiquer dans l'analytique existentiale un manquement non pas à la question du mode d'être de l'instance constituante, mais à celle du mode d'être de ce Dasein qui effectue l'analyse ontologico-existentiale du Dasein facticiel - bref, de « ce Dasein dont Heidegger ne parle pas, à savoir le Dasein qui a écrit $\hat{E}$ tre et temps ${ }^{17}$. Poussée à l'extrême, la contre-objection transcendantale prend en tout cas la forme de l'alternative suivante : soit la validité des descriptions heideggériennes est seulement locale et relève d'une simple Weltanschauung existentielle, soit la démarche théorique de la phénoménologie transcendantale est justifiée.

On comprend à présent pourquoi, dans le texte cité plus haut, Husserl pouvait affirmer que sa propre démarche était «plus primitive » que celle de son ancien assistant. Une fois que les deux phénoménologies sont replacées pour ainsi dire sur l'unique ligne de la théorie philosophique, elles ne se distinguent plus, de fait, que par le degré de conscience réflexive de leur démarche respective, et l'objection heideggérienne peut dès lors être renversée : alors que la phénoménologie husserlienne se voyait reprocher d'en venir trop tardivement à la description de la structure pratique primordiale du monde, et même de ne jamais véritablement l'atteindre, Husserl est à présent en mesure de reprocher à l'analytique existentiale de s'engager quant à elle trop rapidement dans cette description, ou encore d'engager celle-ci «trop haut», c'est-à-dire à un niveau d'analyse qui présuppose la possibilité de sa propre exposition au lieu d'en faire un problème fondamental. Telle est la critique qui se cache derrière l'insis-

\footnotetext{
${ }_{17}$ R. Bernet, «Différence ontologique et conscience transcendantale. La réponse de la Sixième Méditation Cartésienne de Fink", in Husserl, E. Escoubas et M. Richir (éd.), Grenoble, Jérôme Millon, 1989, p.89-116, ici p.95. Cf. S. Luft («Husserl's Concept of the "Transcendental Person" : Another Look at the Husserl-Heidegger Relationship », in International Journal of Philosophical Studies, [en ligne], vol. 13, n², 2005, p. 141-177, ici p. 162).
} 
tance de Husserl à faire de l'esthétique transcendantale la forme primordiale de l'ontologie du monde de la vie, et si Husserl a pu dire (probablement de façon polémique) de cette ontologie qu'elle était la « véritable ontologie fondamentale ${ }^{18}, c^{\prime}$ est au sens où elle déploie la structure apriorique du monde à partir de ce qui ne peut pas ne pas être la strate fondamentale pour une analytique philosophique consciente de son caractère nécessairement théorique.

\section{Le retournement de l'objection d'abstraction et la question de l'anthropologisme}

Venons-en maintenant à l'objection d'abstraction, qui consiste à établir que l'ego transcendantal instauré par la réduction n'est qu'une des possibilités existentiales du Dasein (et pas la plus primitive) abstraitement isolée par Husserl, si bien qu'en toute rigueur, c'est l'analytique existentiale qui devrait fournir son fondement à la phénoménologie constitutive.

La réponse husserlienne à cette objection, telle qu'on la voit se construire dans les manuscrits de recherche, prend cette fois-ci la forme d'un parfait retournement de l'objection contre l'analytique existentiale : si cette dernière dénonce en la subjectivité transcendantale une abstraction du Dasein concret, Husserl soutient à l'inverse que c'est cet existant qu'est le Dasein qui n'est qu'une dimension, qu'une modalité particulière de la subjectivité transcendantale ellemême ${ }^{19}$. L'argument sur lequel repose ce retournement n'est autre que la thèse sur laquelle repose toute sa phénoménologie "tardive », celle de l'identité ou du recouvrement de la subjectivité transcendantale et de l'humanité naturelle. L'idée centrale, à laquelle nous nous limiterons ici, est que le transcendantal et l'humain psychique ou anthropologique ne sont que deux modalités aperceptives distinctes d'une même et unique subjectivité ; tandis que l'humanité naturelle s'aperçoit elle-même comme absolue et primitive et demeure aveugle par principe, il revient à la conversion phénoménologique du regard de rendre visible non seulement la dimension transcendantale de la

\footnotetext{
${ }^{18}$ E. Husserl, Die Krisis der europäischen Wissenschaften und die transzendentale Phänomenologie. Ergänzungsband. Texte aus dem Nachlass, 1934-1937, Dordrecht, Kluwer, 1993, Hua XXIX, p. 151 ; trad. V. Gérard et M. Mavridis in Alter. Revue de phénoménologie, nº 6, 1998, p. 310.

${ }^{19}$ Cf. V. Molchanov, "Husserl and Heidegger: Phenomenology and Ontology", in A.-T. Tymieniecka (ed.), Analecta Husserliana, XXVII, p. 643 sq.
} 
subjectivité $^{20}$, mais par là même aussi le caractère constitué de sa dimension naturelle, anthropologique ou psychique, c'est-à-dire son caractère de "mondanéisation », ou encore d'auto-objectivation mais aussi d'auto-voilement de la subjectivité transcendantale ${ }^{21}$. Il revient donc à l'attitude transcendantale de montrer qu'il appartient par essence au sujet transcendantal de faire l'expérience de soi comme humain, c'est-à-dire dans l'oubli de soi. Malgré les formulations parfois quasi-spéculatives par lesquelles Husserl cherche à la fixer, cette conception revient en définitive à l'idée assez simple selon laquelle le transcendantal n'est rien d'autre que la dimension dans laquelle le naturel est vu comme tel, c'est-à-dire précisément dans sa naïveté constitutive.

Si cette thèse, dont les premières formulations précèdent largement l'année 1927, prend dans le contexte de la controverse avec Heidegger un relief particulier, c'est d'abord parce qu'elle justifie la prétention de la phénoménologie transcendantale à s'emparer thématiquement de l'existence humaine de fait et du monde auquel elle se rapporte concrètement, et à rendre compte par là même du mode d'être de la subjectivité constituante. Si donc les formes sous lesquelles le Dasein humain se rapporte au monde et en détermine le sens ne peuvent être élucidées intégralement que par une philosophie qui reconnaît en l'humanité une détermination de la subjectivité transcendantale, on ne saurait reprocher à la phénoménologie transcendantale de méconnaître le caractère philosophiquement fondamental de la sphère de l'existence humaine concrète dans le monde et les problèmes spécifiques qui s'y posent ; simplement affirme-t-elle que cette concrétude n'est pas immédiatement thématisable comme telle, n'est pas ellemême la mesure de son intelligibilité. Comme l'écrit Husserl dans un manuscrit d'août 1930, il s'agit plutôt de reconnaître que

c'est dans le type d'existence [Dasein] de la réflexion transcendantale qu'est élucidé l'être transcendantal des hommes apparaissant de façon mondaine et du monde même - comme monde environnant dans lequel ils vivent. L'homme installé de la sorte se comprend comme moi transcendantal d'une vie transcendantale d'une certaine structure, constituante, précisément, de l'existence humaine [menschliches Dasein] ${ }^{22}$.

\footnotetext{
${ }^{20}$ Cf. E. Husserl, Cartesianische Meditationen und Pariser Vorträge, La Haye, Martinus Nijhoff, 1950 (désormais : CM), Hua I, §15, Hua I, p. 75 ; trad. M. de Launay, Méditations cartésiennes et Les Conférences de Paris, Paris, PUF, 1994, p. 82.

${ }^{21}$ Cf. E. Husserl, Zur Phänomenologie der Intersubjektivität. Texte aus dem Nachlaß, Dritter Teil: 1928-1935, La Haye, Martinus Nijhoff, 1973 (désormais: ZPI-III), Hua XV, p. 388 sq.; trad. N. Depraz, Sur l'intersubjectivité II, Paris, PUF, 2001, p. 320 sq.

22 E. Husserl, ZPR, Hua XXXIV, p. 154 ; trad. cit. (modifiée), p. 154.
} 
Déjà visible dans la terminologie mobilisée ici par Husserl, la dimension polémique de ces analyses éclate si l'on tient compte du fait que l'identité entre le transcendantal et le mondain peut aussi se décrire en termes d'inclusion réciproque. Ainsi, lorsque Husserl écrit : " chaque homme porte en lui un ego transcendantal», on pourrait croire à la proximité avec Heidegger, c'est-à-dire à la reconnaissance $\mathrm{du}$ fait que le transcendantal n'est qu'une existence mondaine primordiale. Sauf que cette affirmation n'a de sens que corrélée à une autre : " chaque ego transcendantal [...] doit être nécessairement constitué comme un homme dans le monde $\gg^{23}$ - cette deuxième affirmation n'étant pas seulement le complément de la première mais sa condition : car c'est à partir du moment où l'ego humain est su comme ego transcendantal mondanéisé qu'on peut dire qu'il porte en lui le transcendantal comme une de ses possibilités. Par conséquent, isoler comme le fait Heidegger la première affirmation de la seconde et prendre l'homme mondain naturel comme un donné, c'est prendre du dérivé pour du primitif, c'est couper le constitué de son origine et $\mathrm{s}^{\prime}$ engager par définition dans une abstraction qu'on peut désigner si l'on veut du terme d' " anthropologisme », dont la possibilité et l'inévitable absurdité se trouvent conjointement établies. Comme l'écrit Husserl en septembre 1931 :

Le moi transcendantal n'est pas plus dans l'homme (donc dans le monde) que l'homme et le monde sont dans le moi transcendantal [...] si le "dans » désigne un quelconque être-contenu réel. D'un autre côté, il y a bien justement un noyau de vérité faussement interprétée au fondement de cette apparence fondée par l'entrelacement transcendantal de l'humanité et de la subjectivité transcendantale ${ }^{24}$.

L'anthropologisme est donc très exactement une illusion transcendantale et si c'est seulement comme subjectivité transcendantale mondanéisée que la subjectivité humaine peut être thématisée dans sa concrétude, on peut dire que le tort de l'entreprise heideggérienne consiste, au yeux de Husserl, à confondre fondamentalité et absoluité, c'est-à-dire à prendre le caractère fondamental de l'existence factice (et par suite de l'ontologie qui en met les structures au jour) pour un sol philosophique auto-consistant, dont la stabilité n'a pas à être interrogée quant à son origine. Or cela revient à soutenir que l'analytique existentiale se rend au fond coupable d'absolutiser l'attitude naturelle et le monde qui lui est corrélatif: elle est pour Husserl « une fausse

${ }^{23}$ E. Husserl, Krisis, $§ 54$ b), Hua VI, p. 190 ; trad. cit., p. 211-212.

${ }^{24}$ E. Husserl, ZPR, Hua XXXIV, p. 290 ; trad. cit. (modifiée), p. 263. 
philosophie, issue d'une absolutisation d'un monde positiviste (einer positivistischen Welt) $»^{25}$. Cette dernière formule montre que le reproche d'anthropologisme est plus subtil qu'il n'y paraît à première vue, car il ne vise finalement pas tant la notion de Dasein elle-même que l'absolutisation du monde positif à même lequel ce Dasein existe. En d'autres termes, Husserl a peut-être fort bien compris que le Dasein se distingue radicalement de l'humain naturel ; le point décisif est simplement pour lui que cette distinction ne change rien à ce qu'implique l'essentielle intramondanéité de ce Dasein, à savoir la validation naïve de l'être du monde, sur un mode qui ne se distingue en rien, pour le coup, de la façon dont les sciences naturelles de l'humain l'accomplissent pour leur part. L'analytique existentiale serait donc une simple philosophie de l'attitude naturelle, c'est-à-dire une philosophie qui, faute d'accomplir la réduction phénoménologique, demeure prise dans la naïveté constitutive du rapport naturel au monde.

\section{L'intégration transcendantale des Stimmungen: Husserl penseur de l'angoisse et du souci ?}

Parvenu en ce point, il faut faire droit à une objection qui semble s'imposer face à cette lecture husserlienne. Car l'absence de toute réduction explicite ne signifie pas nécessairement une phénoménologie non réductive, condamnée à reconduire le réalisme naïf de l'attitude naturelle. Simplement convient-il d'identifier alors ce qui remplit dans Sein und Zeit cette fonction réductive et, comme on le sait, il revient notamment à Jean-François Courtine d'avoir montré que "l'analyse de l'angoisse [...] constitue comme la "répétition" de la problématique husserlienne de l'épochè et de la réduction phénoménologique-transcendantale $»^{26}$. Or que ce soit le propre de l'angoisse, en tant que Grundstimmung, de prendre en charge la tâche méthodologique de la réduction et d'ouvrir la possibilité d'une description authentiquement phénoménologique du Dasein, c'est un point que Husserl a parfaitement saisi - ce qui témoigne à nouveau de la perspicacité de sa lecture - mais qui ne saurait recevoir son approbation, comme l'indique un manuscrit du printemps 1931 :

La soumission de l'universum de ces actes, pris tous d'un coup, à l'épochè n'engendre plus aucune possibilité d'une poursuite de la vie

\footnotetext{
${ }^{25}$ Ibid., Hua XXXIV, p. 259 ; trad. cit. (modifiée), p. 238.

${ }^{26} \mathrm{~J} .-\mathrm{F}$. Courtine, "Réduction phénoménologique-transcendantale et différence ontico-ontologique », in Heidegger et la phénoménologie, Paris, Vrin, 1990, p. 207-247, ici p. 234.
} 
positionnelle [...] tant que la conscience prédonnant le monde [...] est encore en validité, est encore consciente comme sol de la vie éveillée. Je suis dans l'attitude [Einstellung] naturelle même si je me fige tout à fait dans une angoisse irréfléchie et cesse [einstelle] toute activité, si je ne veux plus rien voir ni entendre, si je ne veux plus recevoir en expérience (orienté vers la connaissance, vers la mise en évidence) ce qui se passe là. Le monde est pourtant là pour moi ; je suis figé, dans un état d'attente figée, pas plus prêt à entrer de nouveau dans une activité mondaine, à diriger des questions vers le monde prédonné, qu'à m'occuper de quoi que ce soit de mondain. Tout comme cet état figé (analogue à une epochè mais qui, contrairement à celle-ci, n'est pas un s'abstenir volontaire), l'épochè universelle peut, sur le sol mondain qui continue à valoir, n'être que provisoirement une manière d'arrêter volontairement la vie positionnelle de monde. Mais il s'agit pourtant là d'un mode, quoique unique en son genre, de la vie mondaine ${ }^{27}$ !

Husserl reconnaît donc à l'angoisse une puissance de suspension, cela ne fait aucun doute. Mais que suspend-elle vraiment ? Les remarques ci-dessus semblent indiquer que loin de suspendre la validité ontologique du monde apparaissant et de révéler par contrecoup son origine, elle me suspend pour ainsi dire au sein du monde, lequel continue à valoir comme sol. C'est moi qui vacille sur le sol du monde et non le monde qui vacille et se dérobe sous mes pieds. À cet égard, on appréciera le jeu de mots sur «einstellen » : dans l'angoisse, j'ai beau cesser [einstellen] tout agir, je n'en suis pas moins engagé [eingestellt] dans le monde au sein duquel mon agir cesse. Bref, si l'angoisse me met en question au sein du monde (jusqu'à mettre éventuellement en question mon être au monde comme tel), elle ne met pas en question l'être du monde lui-même et demeure une façon de se rapporter à lui. En outre, ces lignes montrent que deux caractères interdisent à l'angoisse d'opérer effectivement l'ouverture du champ des phénomènes originaires et de l'ouvrir à une phénoméno-logie, à une science de l'apparaître: elle est involontaire et temporaire. Et si le premier caractère est crucial en ce qu'il montre que la réduction, en tant qu'acte volontaire, possède une dimension éthique pour Husserl, il faut préciser que le second de ces deux caractères est en réalité le plus décisif : en effet une suspension volontaire mais temporaire de la position ontologique de ce qui apparaît est encore possible. Autrement dit, Husserl esquisse dans ces lignes une gradation de trois attitudes suspensives: une suspension involontaire et temporaire (l'angoisse), une suspension volontaire et temporaire (l'épochè de la validité de tous les objets mondains qui ouvre une simple psychologie

\footnotetext{
${ }^{27}$ E. Husserl, ZPR, Hua XXXIV, p. 262-263 ; trad. cit., p. 240 (trad. intégralement corrigée en raison des innombrables contresens qui compromettent l'intelligibilité de ce passage).
} 
phénoménologique) et une suspension volontaire et définitive (l'épochè proprement transcendantale, qui porte sur le monde lui-même comme sol prédonné de toute position et ouvre une phénoménologie transcendantale thématisant les vécus prédonateurs du monde). Bref, le problème de l'angoisse - si l'on ose dire - c'est qu'on en revient (et sans avoir jamais quitté le sol du monde), alors que par opposition à elle, la véritable épochè n'est pas une manière suspensive de se rapporter au monde prédonné mais la suspension de la validité du monde prédonné lui-même, suspension dont on ne revient pas puisqu'une fois qu'elle est accomplie, le regard ne peut plus se porter sur le monde avec la naïveté qui fut le sien auparavant - redevenir naïf étant impossible. Que devient donc l'angoisse dans la phénoménologie husserlienne? Une Stimmung remarquable par son caractère suspensif, mais néanmoins une Stimmung parmi d'autres: on assiste donc à une neutralisation de la critique heideggérienne par l'intégration de l'un de ses concepts fondamentaux à l'analytique intentionnelle. Et cette intégration vaudra pour toute Stimmung dans la mesure où la réduction doit revêtir un caractère volontaire: le caractère affectif de toute Stimmung interdit de reconnaître à aucune d'entre elles une véritable puissance réductive ni, de façon générale, une signification philosophique fondamentale.

C'est ce qu'on peut vérifier aussi dans le cas de la Sorge, du souci. Quelques pages d'un manuscrit inédit de février $1931^{28}$ montrent en effet que de même que dans le cas de l'angoisse, Husserl accepte d'y voir avec Heidegger une Stimmung remarquable mais que rien ne lui semble justifier qu'on en fasse pour autant autre chose que ce qu'elle est, à savoir un vécu affectif parmi d'autres. La spécificité de la Sorge y est récupérée et neutralisée comme simple cas particulier au sein d'une description de l'intentionnalité affective et axiologique. Suivons les quelques paragraphes où se déploie cette analyse. Husserl $s^{\prime}$ interroge sur le caractère axiologique que revêt pour le sujet son monde familier et remarque tout $d$ 'abord que cette évaluation (Wertung) s'accomplit au sein d'une tonalité (Stimmung) d'agrément ou de désagrément. Mais il congédie cette Stimmung au nom de son caractère local : agrément et désagrément se rapportent toujours à tel ou tel lieu, objet, à telle ou telle situation et non au monde dans son ensemble ; elle n'est donc qu'une forme de l'intérêt subjectif, qui est toujours intérêt particulier. Au contraire, l'évaluation globale qu'il

\footnotetext{
${ }^{28}$ E. Husserl, Ms A VI 34 (1931). Une transcription de ce manuscrit est déposée aux Archives Husserl de Paris, à la pagination de laquelle nous renverrons également.
} 


\section{Une réponse husserlienne?}

cherche à décrire n'est pas liée à la Stimmung en ce sens particulier (22a, transcr. p. 33) :

L'évaluation n'est pas tonalité affective en ce sens courant; elle est sentiment de la réussite ou de l'échec mais pas rapporté ainsi à des cas singuliers. L'aspiration humaine en tant qu'aspiration vitale qui, dans l'unité, régit par avance toute aspiration particulière.

L'évaluation qui mérite de recevoir une portée philosophique est donc celle qui se rapporte au tout du monde ambiant, c'est-à-dire qui renvoie corrélativement à la façon dont le sujet se rapporte affectivement et axiologiquement au tout de sa vie. La distinction entre ces deux sens de la Wertung appelle en conséquence une distinction entre deux sens de la Stimmung (22b, transcr. p. 33) :

La tonalité affective que j'obtiens et que $j$ 'ai à chaque fois en tant qu'ego pratique à partir de ma praxis (la satisfaction ou insatisfaction singulière) [...] ; d'un autre côté, la tonalité affective rapportée à tout l'avenir de la vie (également selon l'aspect professionnel ou bien en fin de compte selon tous ses aspects).

Il y a donc une Stimmung correspondant à l'évaluation du tout de la vie comme tel, et si l'on cherche à l'identifier plus précisément, il faut alors tenir compte du caractère structurellement imprévisible du futur (malgré la possibilité d'anticipations justifiées à court terme). D'où la suite du texte (22b, transcr. p. 33-34) :

Il faut tout de suite prêter attention au fait que la vie humaine (dans la forme caractéristique selon laquelle elle est rapportée à un futur et en fin de compte, pour l'homme complet, au tout de la vie) reste toujours dans le flottement de la réussite et de l'échec, et donc toujours dans le danger et le souci; et c'est dans cette mesure qu'il est justifié de désigner la vie du point de vue de sa tonalité affective comme une vie dans le souci de la vie. Le "souci» est quoi qu'il en soit un terme dont le sens renvoie par avance au tout de la vie.

Loin d'être, dans la constitution existentiale du Dasein, la tonalité en laquelle le monde se donne à sa circonspection, le souci devient chez Husserl la tonalité affective selon laquelle la vie subjective se rapporte à ellemême comme à un tout dans son échappement temporel même. On retrouve donc la structure argumentative mise en évidence dans le cas de l'angoisse : il y a bien une spécificité de la Sorge comme Stimmung totale, mais elle n'en reste pas moins un aspect affectif de la vie intentionnelle et ne saurait recevoir pour autant une signification méthodologique eu égard à la possibilité d'une phénoménologie. 


\section{La réélaboration du concept de subjectivité : vers un «Dasein transcendantal»?}

En contrepoint de ces appropriations neutralisantes des analyses heideggériennes, il reste un cas de figure à évoquer, à savoir celui où c'est en réélaborant certains de ses concepts fondamentaux que Husserl parachève sa réponse aux objections de son ancien assistant. L'illustration la plus frappante en est le concept par lequel Husserl cherche à ressaisir l'unité de la subjectivité humaine, dans le recouvrement de l'anthropologique et du transcendantal, et tente ainsi d'élaborer une terminologie destinée à fixer l'alternative phénoménologique et transcendantale à la thématisation heideggérienne de l'existence humaine: il s'agit du concept de "personne transcendantale » (transzendentale Person), dont Sebastian Luft a été le premier à souligner l'importance, malgré la rareté de ses occurrences ${ }^{29}$. Le manuscrit dans lequel cette notion apparaît vraisemblablement pour la première fois date de l'automne 1930 :

Mais le moi transcendantal, entendu concrètement comme subjectivité transcendantale, est précisément la concrétion dévoilée qui coembrasse le sujet humain naturel et, en lui, la personne humaine en son abstraction, qu'il a rendus visibles en cette concrétion par réduction transcendantale. Le moi transcendantal comme pôle et comme substrat de la totalité des pouvoirs est, pour ainsi dire, la personne transcendantale qui vient à institution originaire par la réduction phénoménologique [...]. Il s'avère ici que l'être et la vie naturellement personnels ne sont qu'une forme particulière transcendantale de la vie qui demeure pourtant identique en toutes les autres mutations potentielles, à savoir de la même unité effective et possible de la vie, centrée sur ce même moi-pôle, identique en toutes ces mutations potentielles ${ }^{30}$.

L'introduction du concept de personne transcendantale se situe dans la continuité des analyses du $§ 32$ des Méditations cartésiennes, où la mise en évidence d'habitus au sein du moi transcendantal conduisait Husserl à affirmer que ce moi «qui fonctionne comme centre n'est pas un pôle d'identité vide » mais «qu'il possède un caractère personnel ${ }^{31}$. Si la personne demeure comme telle une notion empirique, le «caractère personnel » est en revanche une structure qui

\footnotetext{
${ }^{29}$ Cf. S. Luft, « Einleitung des Herausgebers », in E. Husserl, Hua XXXIV, p. XXXVI (trad. cit., p. 21) ; "Husserl's Concept of the "Transcendental Person": Another Look at the HusserlHeidegger Relationship », art. cit.

${ }^{30}$ E. Husserl, ZPR, Hua XXXIV, p. 200-201 ; trad. cit., p. 191 (nous soulignons).

${ }^{31}$ E. Husserl, CM, Hua I, §32, Hua I, p. 100 sq.; trad. M. de Launay, Méditations cartésiennes et Les Conférences de Paris, op. cit., p. 113 sq.
} 
peut revenir à l'ego transcendantal et signifier son individualité sans que soit remise en cause sa pureté transcendantale. On voit ainsi que la notion de personne transcendantale vient seulement radicaliser cet effort d'articulation de la personnalité et de la transcendantalité que le passage à une conception génétique de la constitution transcendantale avait rendu possible dès la fin des années dix.

Dans la mesure où il procède de la thèse du recouvrement de l'ego humain et de l'ego transcendantal, on peut bien dire avec Sebastian Luft que le concept de personne transcendantale désigne « l'être humain dans ses dimensions les plus vastes, i. e. intersubjective et génétique, considérées depuis le point de vue de la théorie transcendantale de la constitution ${ }^{32}$. Mais cela signifie qu'il désigne tout aussi bien la subjectivité transcendantale elle-même mondanéisée sous les espèces d'un tel être humain ; comme Husserl l'écrit en décembre 1930 :

Le moi transcendantal n'est rien d'autre que la personne humaine absolue qui, comme telle, apparaît objectivement mais qui, en cette façon objectivée, recèle la possibilité eidétique de l'auto-dévoilement par la réduction phénoménologique ${ }^{33}$.

La personne transcendantale est donc en définitive l'humain concret pour autant qu'on peut dire de lui qu'il est l'instance constituante, et c'est dans cette mesure qu'on peut voir en elle le concept alternatif que la phénoménologie constitutive husserlienne introduit et déploie face au Dasein ontologico-existential pour rendre compte du mode d'être de l'instance constituante. En effet, il n'est pas une caractéristique du Dasein qui ne revienne à la personne transcendantale précisément en vertu de sa personnalité (intramondanéité pratique, relation intersubjective, épaisseur temporelle, habituelle, existentielle, etc.), tandis que tout risque d'absolutisation d'une quelconque donnée mondaine est écarté par principe puisque la personnalité en question n'est alors envisagée que comme transcendantalité personnalisée par sa mondanéisation. C'est pourquoi l'on pourrait dire en somme que le concept de personne transcendantale délimite les contours d'un «Dasein transcendantal »- expression d'autant moins malvenue que Husserl l'emploie lui-même en 1931 pour désigner «l'existence d'une "subjectivité" ([d'une] personnalité individuelle [étant] concrètement dans sa vie) $[\ldots] »^{34}$.

\footnotetext{
32 S. Luft, art. cit., p. 154.

${ }^{33}$ E. Husserl, ZPR, Hua XXXIV, p. 246 ; trad. cit., p. 226.

${ }^{34}$ E. Husserl, ZPI-III, Hua XV, p. 381 : «Sinn des transzendentalen Daseins, Daseins einer „Subjektivität“ (individuellen Personalität konkret in ihrem Leben) [...] ».
} 
Quelles conclusions appellent ces éléments que nous venons de reconstituer sous le titre d'une "réponse husserlienne»? Insistons tout d'abord sur le fait qu'ils vont à l'encontre de l'image convenue d'un Husserl ne parvenant pas à prendre la mesure des objections qui lui sont adressées par son ancien assistant et s'obstinant, en une sorte de fuite en avant, dans un transcendantalisme localement influencé par la phénoménologie existentiale ${ }^{35}$. Les analyses précédentes montrent au contraire que Husserl a parfaitement vu ce que les objections de Heidegger venaient mettre en question au sein de sa propre phénoménologie constitutive et que sur chacun des trois points en question (mode d'être de l'instance constituante, abstraction et théoricisme), sa défense est toujours en même temps polémique, s'attaquant non seulement au statut du discours philosophique de l'analytique existentiale, mais aussi à sa prétention à établir que la transcendantalité n'est qu'une strate de l'humanité concrète.

Reste qu'il est difficile de se défaire du sentiment que l'argumentation polémique de Husserl se situe globalement en deçà des enjeux de l'entreprise heideggérienne, en ce sens qu'elle semble procéder d'un type de philosophie réflexive de la subjectivité dont Heidegger cherche précisément à s'affranchir. Une chose est bien certaine : l'étude minutieuse de l'œuvre de Heidegger à laquelle Husserl s'est livré ne lui a pas permis de saisir dans toute sa subtilité l'articulation de l'analytique du Dasein et de l'élaboration de la question de l'être. Cela signifie entre autres que l'incomplétude de Sein und Zeit n'est restée que formelle aux yeux de Husserl, au sens où elle ne l'a pas empêché de considérer l'ouvrage comme une analytique philosophiquement autonome et auto-consistante du Dasein humain. Dans ces conditions, on peut raisonnablement faire l'hypothèse que Husserl fut d'autant plus sensible aux traits par lesquels l'ouvrage de 1927 se laisse rattacher à une démarche de type transcendantal, au sens le plus large du terme ${ }^{36}$ : recherche de conditions principielles de possibilité (y compris celles d'une philosophie transcendantale elle-même), dégage-

\footnotetext{
${ }^{35}$ Ajoutons qu'ils rendent évidemment superflue toute hypothèse sur le rôle de la «mégalomanie » husserlienne dans cette controverse, contrairement à ce que suggère S. G. Crowell («Does the Husser/Heidegger Feud Rest on a Mistake ? An Essay on Psychological and Transcendantal Phenomenology ", in Husserl Studies, n 18, 2002, p. 123-140, ici p. 123.

${ }^{36}$ Cf. P. Gorner, "Heidegger's Phenomenology as Transcendental Philosophy », in International Journal of Philosophical Studies, vol. 10, n 1, 2002, p. 17-33 ; S. G. Crowell, Husserl, Heidegger and the Space of Meaning, op. cit. Dans la littérature secondaire francophone, cette perspective interprétative a été développée par A. Schnell (De l'existence ouverte au monde fini. Heidegger 1925-1935, Paris, Vrin, 2005), qui en fait l'un des fils conducteurs essentiels d'une lecture qui ne se borne d'ailleurs pas à Sein und Zeit mais s'étend jusqu'à la production heideggérienne du début des années trente.
} 
ment de concepts fondamentaux de type catégoriaux (en l'occurrence, existentiaux), ambition fondationnelle relativement à la scientificité positive. D'où la nature des contre-objections husserliennes, qui s'adressent finalement à la phénoménologie heideggérienne comme une philosophie de la réflexion transcendantale s'adresse à une autre.

Mais quelles sont alors la valeur et la portée philosophiques de ces contre-objections? Car si un quelconque transcendantalisme s'annonce dans les pages de Sein und Zeit, il n'est en rien reconductible à cet idéalisme transcendantal constitutif revendiqué par Husserl, puisqu'on voit bien en effet qu'il est indissociable du problème de l'articulation circulaire de l'analytique existentiale et de l'ontologie fondamentale ainsi que de ce qui deviendra sous la plume de Heidegger la thématique de la "différence ontologique ». Mais précisément, la réticence de Husserl à entendre ce sens inédit du transcendantalisme invite par contrecoup à apercevoir dans ses contre-objections autant de mises en question de cette conjonction du transcendantalisme et de l'herméneutique qui fait assurément la singularité de Sein und Zeit dans le paysage philosophique du XXe siècle. En d'autres termes, la valeur philosophique des arguments husserliens pourrait venir de ce qu'ils mettent en lumière les limites d'un transcendantalisme partiel, ne s'assumant pas tel quel intégralement, ou encore l'incompatibilité de principe de la phénoménologie, comme philosophie du donné comme tel, avec l'herméneutique : en pointant un défaut de fondation réflexive dans la démarche de son ancien assistant, il n'est pas impossible que Husserl ait cherché à montrer qu'un transcendantalisme qui prétend faire l'économie d'une égologie transcendantale en lui substituant la circularité herméneutique de la compréhension se condamne en réalité à manquer de radicalité fondative, c'est-à-dire à ne pas parvenir à se débarrasser de toute naïveté dans son élucidation de ce que Heidegger nomme l'« existence moyenne ». Ainsi ressaisies, les contre-objections par lesquelles Husserl stigmatise les insuffisances de la phénoménologie qui se déploie dans Sein und Zeit ne peuvent pas être considérées comme inconsistantes. Au contraire, on peut même aller jusqu'à suggérer qu'elles ne sont pas foncièrement étrangères aux raisons pour lesquelles Heidegger lui-même n'a pas tardé, après sa publication, à juger insatisfaisante la démarche de l'ouvrage de 1927. 